\title{
SPLITTING FIELDS AND SEPARABILITY
}

\author{
MARK RAMRAS ${ }^{1}$
}

\begin{abstract}
It is a classical result that if $(R, \mathfrak{M})$ is a complete discrete valuation ring with quotient field $K$, and if $R / \mathfrak{M}$ is perfect, then any finite dimensional central simple $K$-algebra $\Sigma$ can be split by a field $L$ which is an unramified extension of $K$. Here we prove that if $(R, \mathfrak{M})$ is any regular local ring, and if $\Sigma$ contains an $R$-order $\Lambda$ whose global dimension is finite and such that $\Lambda / \operatorname{Rad} \Lambda$ is central simple over $R / \mathfrak{M}$, then the existence of an " $R$-unramified" splitting field $L$ for $\Sigma$ implies that $\Lambda$ is $R$-separable. Using this theorem we construct an example which shows that if $R$ is a regular local ring of dimension greater than one, and if its characteristic is not 2 , then there is a central division algebra over $K$ which has no $R$-unramified splitting field.
\end{abstract}

In the study of central simple algebras, splitting fields play an important role. A well-known classical result states that if $\Sigma$ is a finite dimensional central simple algebra over a field $K$, then $\Sigma$ can be split by a field $L$ which is a finite separable extension of $K$. If $K$ is the quotient field of a complete discrete valuation ring $R$ whose residue class field $R / \mathfrak{M}$ is perfect, then $L$ can be chosen so that the integral closure, $S$, of $R$ in $L$, is $R$-separable [4, Chapter 5, Theorem 8]. Using this fact, Auslander and Goldman [2, Proposition 8.4] proved that if $R$ is a discrete valuation ring (not necessarily complete) with perfect residue class field and if $\Lambda$ is a maximal $R$-order in $\Sigma$ such that the center of $\Lambda / N$ is $R / \mathfrak{M}$ (where $N$ is the Jacobson radical of $\Lambda$ ), then $\Lambda$ is $R$-separable. Our purpose in this paper is to generalize this result, and thereby produce an example of a division algebra $\Sigma$ which cannot be split by any " $R$-unramified" extension $L$ of $K$, where $R$ is a regular local ring of dimension greater than one.

Definition. Let $R$ be a domain with quotient field $K$. A finite field extension $L$ of $K$ is $R$-unramified if $L$ is the quotient field of some $R$-algebra $S$ which is $R$-separable and finitely generated as an $R$-module.

Received by the editors May 8, 1972.

AMS (MOS) subject classifications (1970). Primary 16A16, 16A40, 13A20.

Key words and phrases. Central simple algebra, splitting field, separable, $R$-unramified, maximal order, global dimension.

${ }^{1}$ This research was partially supported by the U.S. Army Research Office (Durham). 
THEOREM. Let $(R, \mathfrak{M})$ be a regular local ring with quotient field $K$. Let $\Sigma$ be a finite dimensional central simple $K$-algebra which contains an $R$-order $\Lambda$ such that $\mathrm{gl} \operatorname{dim} \Lambda<\infty$, and $\Lambda / \operatorname{Rad} \Lambda$ is central simple over $R / \mathfrak{M}$. Suppose $\Sigma$ has an $R$-unramified splitting field $L$. Then $\Lambda$ is $R$-separable.

Proof. Assume first that $R$ is complete. By hypothesis, $L$ is the quotient field of $S$, an $R$-separable domain which is a finitely generated $R$ module. According to [1, Proposition 4.3], $S$ is $R$-free. Since $R$ is complete, so is $S$, and therefore any proper idempotent in $S / \operatorname{Rad} S$ could be lifted to one in $S$. But $S$ is a domain, and hence contains no proper idempotent, so $S / \operatorname{Rad} S$ has none either and is therefore a field. Since $S$ is $R$-separable, $\operatorname{Rad} S=\mathfrak{M} S$. Hence $S$ is local, with maximal ideal $\mathfrak{M} S$. It follows that $S$ is a regular local ring.

Since $S / \mathfrak{M} S$ is a field, $S / \mathfrak{M} S \otimes_{R / \mathfrak{M}} \Lambda / N$ is central simple over $S / \mathfrak{M} S$, where $N=\operatorname{Rad} \Lambda$. But $S / \mathfrak{M} S \otimes_{R / \mathfrak{M}} \Lambda / N \approx S \otimes_{R} \Lambda / N$, so the latter, in particular, is a simple ring. Since $S$ is $R$-free, the sequence

$$
0 \rightarrow S \otimes_{R} N \rightarrow S \otimes_{R} \Lambda \rightarrow S \otimes_{R} \Lambda / N \rightarrow 0
$$

is exact. Hence $S \otimes_{R} N$ is a maximal two-sided ideal in $S \otimes_{R} \Lambda$. Furthermore, since $N=\operatorname{Rad} \Lambda$ and $S \otimes_{R} N$ is a two-sided ideal, $S \otimes_{R} N \subset$ $\operatorname{Rad}\left(S \otimes_{R} \Lambda\right)$. Thus $S \otimes_{R} N=\operatorname{Rad}\left(S \otimes_{R} \Lambda\right)$ and $S \otimes_{R} \Lambda$ is quasi-local (i.e. modulo its radical it is a simple ring). By [2, Theorem 1.8], gl $\operatorname{dim} S \otimes_{R}$ $\Lambda \leqq \mathrm{gl} \operatorname{dim} \Lambda<\infty$. Thus $S \otimes_{R} \Lambda$ has finite global dimension and is a quasilocal $S$-order in $L \otimes_{K} \Sigma$, a full matrix ring over $L$. We have recently proved [3, Theorem 4] that under these conditions $S \otimes_{R} \Lambda$ is a full matrix ring over $S$ and is therefore $S$-separable. Since $S$ is $R$-separable, it follows from [2, Theorem 2.3] that $S \otimes_{R} \Lambda$ is $R$-separable. But $S$ is $R$-free, so by [2, Proposition 1.7], $\Lambda$ is $R$-separable.

Now, for the general case, let $(R, \mathfrak{M})$ be any regular local ring and let $(\hat{R}, \hat{\mathfrak{M}})$ be its completion in the $\mathfrak{M}$-adic topology. Let $\hat{K}$ be the quotient field of $\hat{R}$, let $\hat{\Lambda}=\Lambda \otimes_{R} \hat{R}$, and let $\hat{\Sigma}=\Sigma \otimes_{K} \hat{K}$. Finally, let $\hat{L}=L \otimes_{K} \hat{K}$ and $\hat{S}=S \otimes_{R} \hat{R}$. Since $L$ is $K$-separable, $\hat{L}$ is $\hat{K}$-separable, and therefore $\hat{L}=L_{1} \oplus \cdots L_{s}$ (ring direct product) where each $L_{i}$ is a separable field extension of $\hat{K}$. If $S_{i}$ denotes the image of $\hat{S}$ in $L_{i}$ under the projection map $\hat{L} \rightarrow L_{i}$, then $\hat{S}=S_{1} \oplus \cdots \oplus S_{s}$. Thus $S_{i}$ is an $\hat{R}$-separable domain with quotient field $L_{i}$. Since $L$ splits $\Sigma, \hat{L}$ splits $\hat{\Sigma}$. Hence for each $i, L_{i}$ splits $\hat{\Sigma}$. If $\hat{N}=N \Theta_{R} \hat{R}$, then $\hat{N}=\operatorname{Rad} \hat{\Lambda}$, and $\hat{\Lambda} / \hat{N} \simeq \Lambda / N$. Hence $\hat{\Lambda} / \hat{N}$ is central simple over $\hat{R} / \hat{\mathfrak{M}}$. Also, $\mathrm{gl} \operatorname{dim} \hat{\Lambda}=\mathrm{gl} \operatorname{dim} \Lambda$. We may conclude, by our proof for the complete case, that $\hat{\Lambda}$ is $\hat{R}$-separable. But this implies that $\Lambda$ is $R$-separable.

Notation. For a commutative ring $A, B(A)$ will denote the Brauer group of $A$ (cf. [2]). 
COROLlARY. Let $(R, \mathfrak{M})$ be a regular local ring with quotient field $K$ and assume that $B(R / \mathfrak{M})=0$ (e.g. $R / \mathfrak{M}$ might be algebraically closed). Let $\Lambda \neq R$ be a central $R$-algebra, finitely generated as an $R$-module. Suppose that $\Lambda$ is local and $\mathrm{gl} \operatorname{dim} \Lambda<\infty$. Then $\Lambda \otimes_{R} K$ is a central division algebra over $K$ which has no $R$-unramified splitting field. (If $R / \mathfrak{M}$ is perfect, then the dimension of $R$ is greater than one.)

Proof. $\Lambda \otimes_{R} K$ is an artin ring containing a local order with finite global dimension. By [3, Corollary 2], $\Lambda \otimes_{R} K$ is a division ring. Now if $\Lambda \otimes_{R} K$ has an $R$-unramified splitting field, then by the preceding theorem $\hat{\Lambda}$ is $\hat{R}$-separable. Since $\hat{R}$ is complete, the map $B(\hat{R}) \rightarrow B(R / \mathfrak{M})$, induced by the projection map $\hat{R} \rightarrow \hat{R} / \hat{\mathfrak{M}}\}=R / \mathfrak{M}$, is a monomorphism [2, Corollary 6.2]. Since $B(R / \mathfrak{M})=0$, it follows that $B(\hat{R})=0$. Hence for some integer $q$, $\hat{\Lambda} \simeq M_{q}(\hat{R})$, the ring of $q \times q$ matrices over $\hat{R}$. Therefore $\Lambda / \operatorname{Rad} \Lambda \simeq$ $\hat{\Lambda} / \operatorname{Rad} \hat{\Lambda} \simeq M_{q}(R / \mathfrak{M})$. But since $\Lambda$ is local, $\Lambda / \operatorname{Rad} \Lambda$ is a division ring. Hence $\Lambda / \operatorname{Rad} \Lambda \simeq R / \mathfrak{M}$ and $q=1$. Thus $\hat{\Lambda}=\hat{R}$ and so $\Lambda=R$. Contradiction. Thus $\Lambda \otimes_{R} K$ has no $R$-unramified splitting field.

Since $\hat{R}$ and $\hat{\Lambda}$ share all the properties of $R$ and $\Lambda, \hat{\Lambda} \otimes_{\hat{R}} \hat{K}$ has no $\hat{R}$ unramified splitting field. Thus if $R / \mathcal{M N}$ is perfect, $\hat{R}$ cannot be a discrete valuation ring [4, Chapter 5, Theorem 8]. Hence $\mathrm{gl} \operatorname{dim} R=\mathrm{gl} \operatorname{dim} \hat{R}>1$.

We conclude with an

EXAMPLE. Let $(R, \mathfrak{M})$ be a regular local ring of dimension 2 , and let $\mathfrak{M}=\left(X_{1}, X_{2}\right)$. Assume that $K$, the quotient field of $R$, has characteristic $\neq 2$. Let $\Sigma$ be the quaternion $K$-algebra $K[1, \alpha, \beta, \alpha \beta]$ defined by $\alpha^{2}=$ $X_{1}, \beta^{2}=X_{2}$, and $\beta \alpha=-\alpha \beta$. Then $\Sigma$ has no $R$-unramified splitting field.

Proof. Let $\Lambda=R[1, \alpha, \beta, \alpha \beta] . \Lambda$ is an $R$-order in $\Sigma$. $\operatorname{Rad} \Lambda=\Lambda(\alpha, \beta)$, so that $\Lambda / \operatorname{Rad} \Lambda \simeq R / \mathfrak{M}$. Since

$$
0 \longrightarrow \Lambda\langle\beta, \alpha\rangle \longrightarrow \Lambda \oplus \Lambda \stackrel{(\alpha, \beta)}{\longrightarrow} \Lambda(\alpha, \beta) \longrightarrow 0
$$

is a minimal free $\Lambda$-resolution of $\operatorname{Rad} \Lambda$, the projective dimension of $\operatorname{Rad} \Lambda$ is 1 , and hence $\mathrm{gl} \operatorname{dim} \Lambda=2$.

Since $\operatorname{Rad} \Lambda=\Lambda(\alpha, \beta) \supsetneqq \Lambda\left(X_{1}, X_{2}\right)=\mathfrak{M} \Lambda$, it follows that $\operatorname{Rad} \Lambda \neq I \Lambda$ for any ideal $I$ of $R$. Hence $\Lambda$ is not $R$-separable [2, Corollary 3.2] and thus by our theorem, $\Sigma$ has no $R$-unramified splitting field.

It should be noted that in the above example we restricted the global dimension of $R$ to 2 for notational convenience only. If $\mathrm{gl} \operatorname{dim} R=n \geqq 3$ and $\mathfrak{M}=\left(X_{1}, \cdots, X_{n}\right)$ and if $\Lambda$ is defined as above, then the only difference is that $\operatorname{Rad} \Lambda=\Lambda\left(\alpha, \beta, X_{3}, \cdots, X_{n}\right)$ and $\mathrm{gl} \operatorname{dim} \Lambda=n$.

\section{REFERENCES}

1. M. Auslander and D. A. Buchsbaum, On ramification theory in noetherian rings, Amer. J. Math. 81 (1959), 749-765. MR 21 \#5659. 
2. M. Auslander and $O$. Goldman, The Brauer group of a commutative ring, Trans. Amer. Math. Soc. 97 (1960), 367-409. MR 22 \#12130.

3. M. Ramras, Orders with finite global dimension, Pacific J. Math. (to appear).

4. O. F. G. Schilling, The theory of valuations, Math. Surveys, no. 4, Amer. Math. Soc., Providence, R.I., 1950. MR 13, 315.

Department of Mathematics, Boston College, Chestnut Hill, Massachusetts 02167 